\title{
Face detection in image sequences using a portable thermal camera
}

\author{
by A. Kwaśniewska*, J. Rumiński** \\ * Gdansk Univ. of Technology, 80-233, Narutowicza 11/12, Gdansk, Poland, kwasniewskaala@gmail.com \\ ** Gdansk Univ. of Technology, 80-233, Narutowicza 11/12, Gdansk, Poland, jacek.ruminski@pg.gda.pl
}

\begin{abstract}
Face detection is often a first step in quantitative analysis of face images. It is an important research area for visible images and recently also for thermography. Due to technological developments thermal cameras may be embedded into wearable devices to provide remote healthcare. In this paper, we compared three algorithms for face detection in thermal images by testing execution time, accuracy, symmetry ratio and false-positives. We investigated whether the computational performance of the methods allows for reliable face detection in real-time. The results show that the best algorithm allows to detect faces with acceptable accuracy $(90.5 \pm 4.34 \%)$ in about $23 \mathrm{~ms}$ per frame.
\end{abstract}

\section{Introduction}

Recently, vision-based tracking systems have become very popular because of many possible use cases. Exemplary applications vary from solutions based on biometrics, such as identifying or verifying identities to solutions applied in medicine, like estimation of the respiration rate using an analysis of temperature changes in area of a nose. In systems of this kind face detection has to be a first step [1].

Most of researches are focused on face recognition in visible images, but recently more and more effort has been spent on using infrared thermal imaging. This fast growth in popularity is caused by the fact that utilization of advantages poses by processing thermal images may turned out to be really beneficial. First of all, infrared thermal images are insensitive to changes in illumination conditions, while in visible imagery this parameter may significantly affect the image information [2]. Secondly, in infrared imagery the temperature distribution is recorded, so the image information does not depend on skin colour or other appearance parameters. On the other hand, geometric and appearance features in thermal images are more blurred than in visible images. Therefore, using infrared imagery may turn out to be a more challenging task. Some solutions described in literature propose to manually mark interested region [3] or first detect it in associated visible image and then apply to thermal images [4]. Yet, for medical applications a first priority is to design fully automatic system which can work in real time, so the need for creating a system of this kind continues to be a challenging area of research [5].

Moreover, many studies on using wearable devices in medicine have already been carried. Some examples include patient identification by using specially prepared graphical markers [6] [7] or face recognition [8]. Furthermore, because of technological development thermal cameras have lately become much smaller. Taking it into account, another idea is to use thermal camera embedded in smart glasses to process thermal images and estimate respiration rate of a patient. However, usually small thermal cameras suffer from relatively poor image quality, so this task may be rather complicated and should be carefully examined.

In this paper, we investigated whether the computational performance of the three analysed methods allows for reliable, automatic, real-time face detection from thermal images of poor quality. In our experiments, we compared execution time, accuracy, symmetry ratio and false-positives (detected false cases and wrong areas). The rest of the paper is structured as follows: Section II presents existing solutions and methods for face detection. Section III describes algorithms that were used to detect face. In Section IV we demonstrate the experimental results for face detection which include time processing, accuracy defined by displacement of a centre of a face in detected region, symmetry of detected area and percentage of recognized false positives. In Section $V$ we discuss the results, present some ideas of using face detection in medicine and finally we conclude the paper.

\section{Related work}

Face detection from images is an important step in many image-based recognition systems. Exemplary applications include tracking and recognizing identities automatically (user authentication, airport security checks and documents with included biometric information) [5] or systems introduced in medicine, for example in ophthalmology, dermatology, stomatology, laryngology and neurology [9].

Especially in medical applications of thermography location of the face and facial features are very useful. Some examples include the non-contact estimation of the respiration rate using an analysis of temperature changes in the nostril region [10] [11]. In the proposed approach [10] the region affected by respiration process is marked in the center of a nose tip. Then, in order to compute the respiration rate feature extraction and signal processing methods were 


\subsection{1/qirt.2016.071}

applied to this region. Another application of thermography in medicine allows for differential analysis of vascularization in the evaluation of facial paralysis [12]. The presented method focuses on facial feature localization based on temperature distribution specificity and edge detection. Actual results of performed experiments show that automatic assessment of facial nerve function is possible by using infrared thermal imagery.

Moreover, because of thermal image processing development, a lot of medical procedures may be performed in much shorter time. One of the systems described in literature allows to detect the fetal face from the 3D data in 1 second on a dual-core $2.0 \mathrm{GHz}$ computer, so not only does it achieve excellent precision but also runs very fast [13]. By utilization of a boosting-based 2D profile detection this system allows to speed up a standard procedure, which is rather cumbersome and time-consuming even for expert sonographers, because it requires to manually search for the optimal view of the fetal face in 3D ultrasound volumes.

Because of fast technology development, benefits may be realized by combining the idea of face detection system with wearable technology. This combination may be possible because of the fact that recently the availability of small thermal camera modules has allowed to embed them in wearable devices like smart glasses. For example, a physician specializing in treatment of allergies could observe a patient and analyze the breath patterns calculated for the automatically detected nostril region [11]. Providing a tool for initial diagnosis, remote patient monitoring or patient identification in a portable device may speed up medical procedures which include saving, comparing or getting information about patients.

Many face detection methods have been proposed in literature. For visible light images, the Viola-Jones face detection framework [14] is often used since it is robust and it works practically in real time. This method consists of 2 parts: training and detection phase. Usually a training is rather slow, but a classifier learned from labelled examples allows for real - time object detection. The recognition process is more efficient because of taking advantage of the Haar-like features, which allow to encode the presence of oriented contrasts between two regions [15]. The features which can be computed in a short time irrespectively of their position [16] are presented in Fig. 1. Recently an improvement to standard Haar Feature-based classifier was also proposed [17]. This technique known as AdaBoost algorithm allows to shorten the computation time while preserving accuracy similar to single stage procedure which is more complex [10]. This improvement is possible because of reduction of all irrelevant features. The OpenCV implementation of Haar Feature-based Cascade Classifiers combined with AdaBoost algorithm has around 6000 Haarlike features, while a standard method is based on more than $160000+$ features.

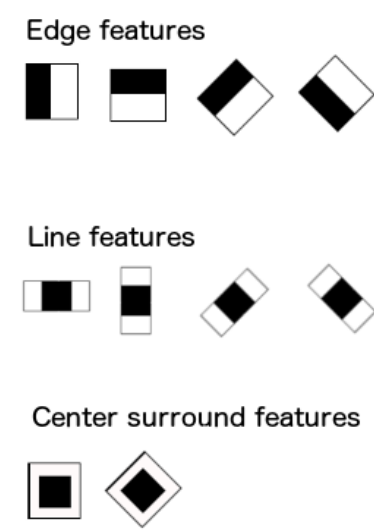

Fig. 1. Examples of Haar - like features

Although many researches have been conducted for face detection in visible images, there is also a need to spend some effort on making realistic use of infrared imagery as it poses some advantages over image recognition in visible light. Infrared thermal images are insensitive to changes in illumination conditions, so in comparison to visible images they can be employed in uncontrolled environments [18]. Moreover, thermal infrared (IR) images record the temperature distribution, so they are independent on appearance parameters [19]. Yet, detecting face and facial features in thermography is very challenging and complex, because geometric and appearance features in thermography are more blurred than in visible images [18].

Face detection from thermal images is usually based on multistep procedures, which use thresholding operations [20], Haar wavelet transform (HWT) or Local Binary Pattern (LBP) [5]. Often methods for the detection of characteristic areas in thermal face images are also proposed [9] [21]. Some of them take advantage of brightness (temperature) distribution or predefined face symmetry patterns [22]. The thresholding operation utilizes the fact that in the thermal images a face has a highest content than the rest of the image. Taking it into account, an interesting area can be easily extracted by detecting higher intensities.

Unfortunately, in many related papers, details about time performance or, in general, computational performance analyses are not presented. Taking it into account there is a need to determine whether analyzed methods allow for reliable real-time face detection. Because of high efficiency of the Viola-Jones algorithm for visible images, we decided to train similar classifier using thermal test images and to analyze the results in comparison with two simple (and 


\subsection{1/qirt.2016.071}

not perfect) face detection algorithms. Additionally, in the context of practical application for smart glasses, we used a portable and mobile camera with relatively poor image quality.

\section{Methodology}

Recently, real-time face detection algorithms have garnered tremendous attention, because of a wide range of possible use cases [23]. Exemplary applications vary from biometrics solutions [5] to solutions applied in different medical areas [9]. Although many methods which facilitate fast processing and high accuracy have already been described [14], an automatic reliable real-time face recognition is still a challenging task.

Furthermore, most of existing solutions are primarily concerned with face detection in visible images. However, benefits may be also realized by analysing thermal images, as they could compensate some disadvantages of visible spectrum images. For example, thermal infrared (IR) images, are insensitive to changes in illumination conditions [18] and emissivity values of the IR spectrum are quite homogeneous for different skin colors [19].

In the view of foregoing, three face detection methods were analyzed on the set of thermal images: The ViolaJones algorithm (VJ), the pattern-matching (PM) algorithm, and the method based on active-contour (AC) detection. In experiments the Tamarisk 320 long wave thermal camera module was used (resolution $320 \times 240$, sensitivity $<50 \mathrm{mK}$, size $3 \mathrm{~cm} \times 2.6 \mathrm{~cm} \times 4 \mathrm{~cm}$ ) to acquire images. Created set consist of 12000 positive cases (face thermal images) and 3000 false cases (thermal images of other objects). Positive cases (face thermal images) were acquired from video sequences recorded from 19 volunteers (age: $23.7 \pm 5.2 ; 7$ men, 12 women). Example images collected during experiment are presented in Fig. 2.
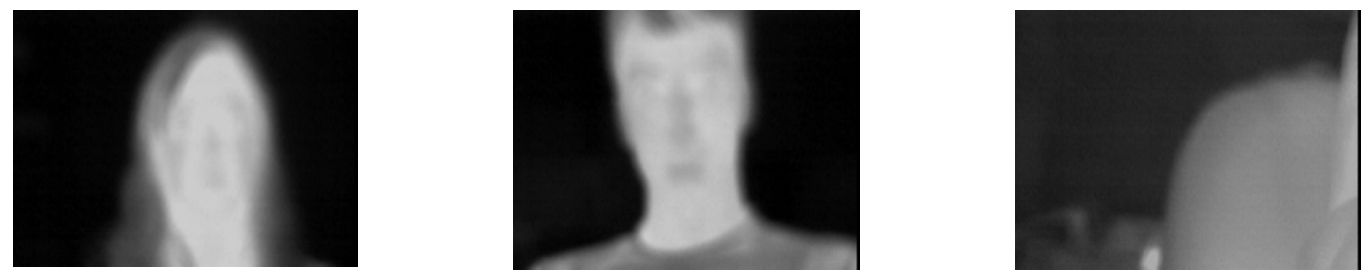

Fig. 2. Examples of acquired thermal images, from the left: Female 22 years, male 23 years (positive cases), different object (negative case)

\subsection{Face detection algorithms}

The aim of our research was to determine whether computation performance of presented algorithms allows to recognize face in the real time. The Viola - Jones algorithm is often used for visible light images due to high computation performance and precision. However, facial features for thermal images are not the same as for visible light images. Therefore, we decided to check whether similar classifier with specially prepared cascaded structure, learned from labelled training thermal images allows to achieve comparable accuracy and efficiency. Using positive and negative cases we trained 12 cascade stages with OpenCV library. In each stage we defined a maximum acceptable error by setting a maximum false alarm to 0.6 . It means that the stage was interrupted when the face was detected in $60 \%$ of negative samples. The result of the training phase was the Haar-based feature classifier that was used for face detection in the test data set.

Usually, a first step in a majority of methods for face detection in thermal images is thresholding. This operation utilizes the fact that in the thermal images a face has a highest content than a background. Taking it into account, an image can be easily extracted into a foreground and a background by detecting higher intensities which represent the face. To facilitate subsequent steps of $P M$ and $A C$ algorithms, the thresholding technique was applied as a preprocessing phase. Then, in the PM method we iterated through image pixels and compared the overlapped patches against the prepared template using the 'CV_TM_SQDIFF' method from the OpenCV library defined as Eq. (1), where I denotes an image, $R$ a result and $T$ a template.

$$
R(x, y)=\sum_{x^{\prime}, y^{\prime}}\left(T\left(x^{\prime}, y^{\prime}\right)-I\left(x+x^{\prime}, y+y^{\prime}\right)\right)^{2}
$$

The best match was found as a global minimum from all comparison results. The location of the best match was used to draw a rectangle on the face area.

In the AC algorithm after applying threshold, we were looking for farthest edge points, which represent edge points of a facial contour. Then, the vertical lines were led through coordinates of the left and right point and horizontal lines were led through coordinates of the top and down points. The center of gravity of the resulting rectangle was calculated and used to inscribe an ellipse shape in the rectangle which matched the face contour. The described steps are presented in Fig. 3. 


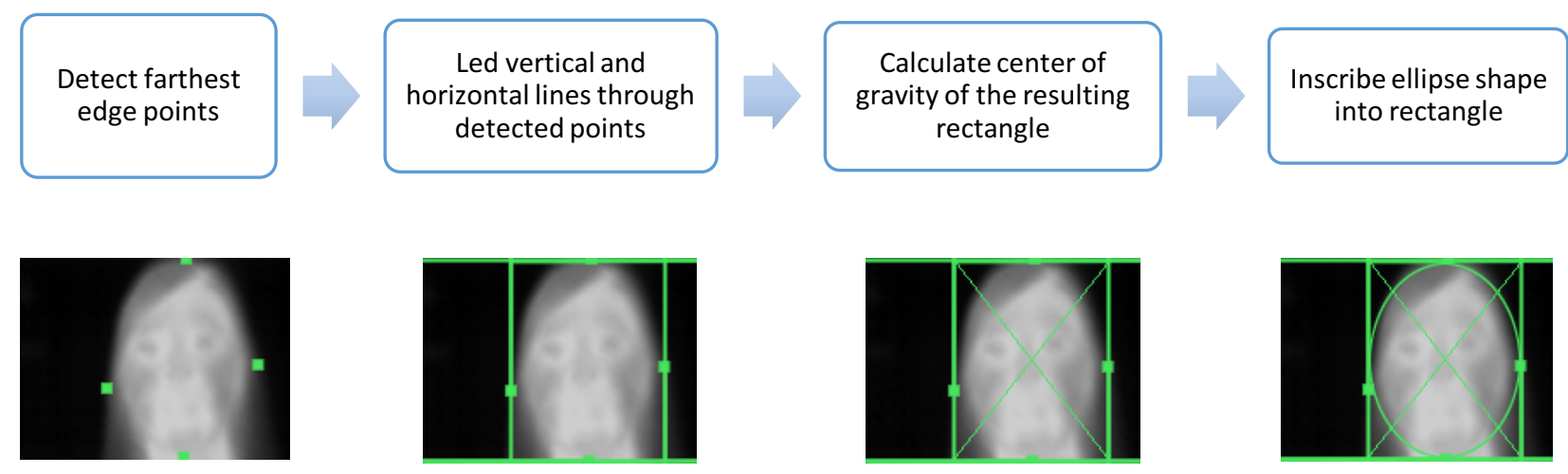

Fig. 3. Matching ellipse shape into face contour in AC algorithm

\subsection{Analysis of presented methods}

Our research underlines the importance of real-time reliable face detection in thermal imagery. In performed experiments we investigated whether the computational performance of the presented methods allows to achieve it. All algorithms were tested for execution time, accuracy, symmetry ratio, false positives (for detected false cases and wrong areas) on the set of 60 test images that portray male and female faces (10 images for each person from 6 volunteers test group). Test images were different than images used to train the cascade. During the experiment each volunteer was asked to remain in quiescence while being positioned with the face placed toward camera.

Taking into account the geometrical symmetry and proportion of a face [24], a symmetry center was marked in the center of a nose as presented in Fig. 4. This manually indicated point was used to analyse the symmetry of the detected facial region. Additionally, the accuracy was measured as a distance between a center of gravity of the area detected by algorithm and the symmetry center marked manually, divided by the face height.

We also measured influence of template scaling in the PM method. For this reason, we created the Laplacian pyramid of the original template (3 levels). Then, we expected to find a match to each level in the face image of original size. Moreover, in order to decide whether algorithms could be used in real-time applications, we examined the computation performance of all algorithms by calculating execution time required to process one frame. All experiments were performed on the MacBook Air computer with $1,6 \mathrm{GHz}$ Intel Core i5 processor (8 GB RAM).
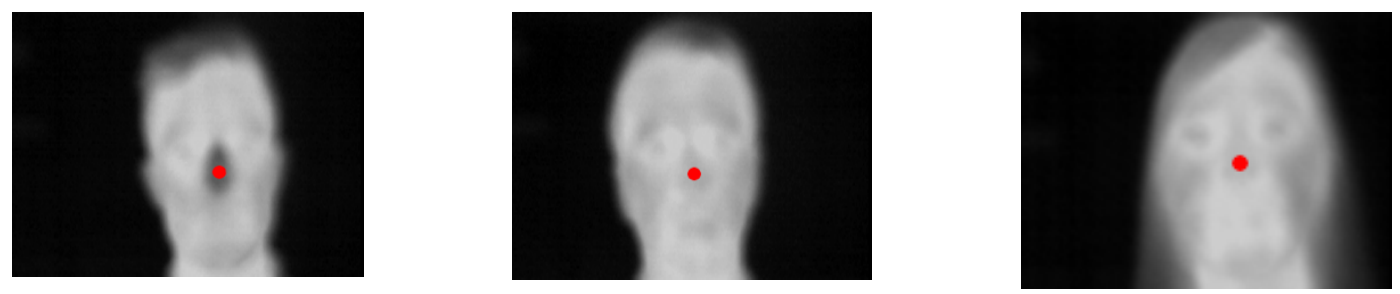

Fig. 4. Manually marked symmetry center in the center of a nose (red dot)

\section{Results}

In the Table 1, 2 and 3 results of face detection algorithms executed for test images are presented and compared. For all positive test images the number of false positives was 0 . However, additional test set of negative images was used to analyze the number of false positives for all 3 algorithms. The results show that the VJ algorithm detected faces in $25 \%$ of negative test images and the AC algorithm in $95 \%$ of negative test images. The PM algorithm produced 0 false positives.

In Fig. 5 the examples of face detection phase applied on the same frame using the Viola-Jones, the patternmatching and the active-contour algorithms are illustrated. 
Table 1. Average processing time - the results for VJ, PM and AC algorithm

\begin{tabular}{|l|l|l|l|}
\hline & \multicolumn{3}{l|}{ Average time $[\mathrm{ms}]$} \\
\hline Subject & VJ & PM & AC \\
\hline Female 23 years & $31 \pm 3.74$ & $62 \pm 13.79$ & $60 \pm 1.87$ \\
\hline Male 44 years & $26 \pm 7.64$ & $36 \pm 2.77$ & $59 \pm 1.41$ \\
\hline Male 25 years & $21 \pm 2.74$ & $40 \pm 8.92$ & $58 \pm 4.09$ \\
\hline Female 22 years & $19 \pm 2.5$ & $47 \pm 12.6$ & $57 \pm 4.6$ \\
\hline Male 24 years & $20 \pm 4.09$ & $38 \pm 5.7$ & $61 \pm 2.44$ \\
\hline Female 23 years & $18 \pm 3.03$ & $33.8 \pm 1.92$ & $58 \pm 2.91$ \\
\hline
\end{tabular}

Table 2. Accuracy - the results for VJ, PM and AC algorithm

\begin{tabular}{|l|l|l|l|}
\hline & \multicolumn{2}{|l|}{ Accuracy [\%] } \\
\hline Subject & VJ & PM & AC \\
\hline Female 23 years & $87 \pm 0.02$ & $88 \pm 0.03$ & $91 \pm 0.04$ \\
\hline Male 44 years & $83 \pm 0.03$ & $87 \pm 0.04$ & $88 \pm 0.05$ \\
\hline Male 25 years & $86 \pm 0.02$ & $89 \pm 0.06$ & $92 \pm 0.03$ \\
\hline Female 22 years & $96 \pm 0.04$ & $98 \pm 0.01$ & $91 \pm 0.07$ \\
\hline Male 24 years & $97 \pm 0.01$ & $94 \pm 0.04$ & $93 \pm 0.02$ \\
\hline Female 23 years & $91 \pm 0.01$ & $94 \pm 0.02$ & $84 \pm 0.07$ \\
\hline
\end{tabular}

Table 3. Symmetry ratio - the results for VJ, PM and AC algorithm

\begin{tabular}{|l|l|l|l|}
\hline \multicolumn{4}{|l|}{ Symmetry ratio } \\
\hline Subject & VJ & PM & AC \\
\hline Female 23 years & $0.75 \pm 0.02$ & $0.55 \pm 0.01$ & $0.98 \pm 0.01$ \\
\hline Male 44 years & $0.73 \pm 0.04$ & $0.92 \pm 0.03$ & $1.14 \pm 0.06$ \\
\hline Male 25 years & $0.77 \pm 0.14$ & $1.05 \pm 0.07$ & $1.16 \pm 0.09$ \\
\hline Female 22 years & $0.8 \pm 0.02$ & $0.97 \pm 0.03$ & $1.25 \pm 0.05$ \\
\hline Male 24 years & $0.66 \pm 0.03$ & $0.66 \pm 0.01$ & $0.63 \pm 0.03$ \\
\hline Female 23 years & $0.58 \pm 0.03$ & $0.85 \pm 0.12$ & $0.45 \pm 0.01$ \\
\hline
\end{tabular}
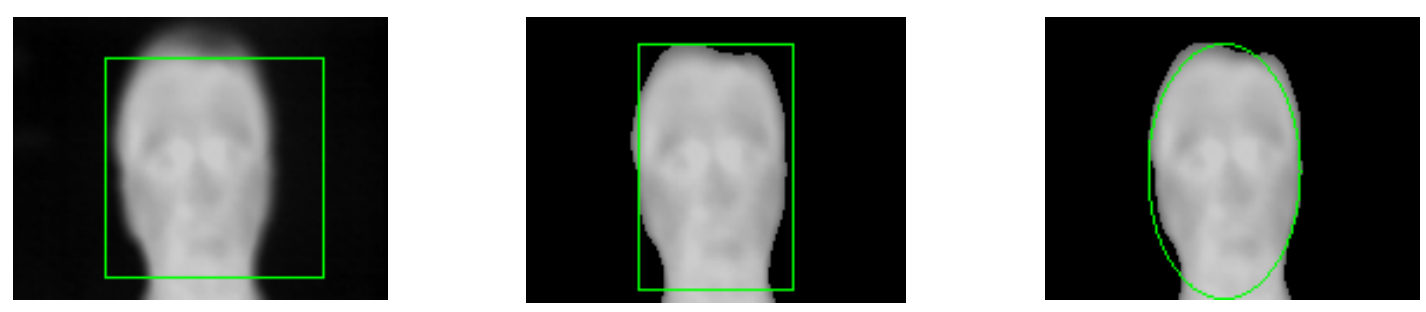

Fig. 5. From the left: face detected with VJ, PM and AC algorithms

\section{Discussion and conclusion}

Analysis performed for various face detection algorithms showed that it is possible to recognize face in real time with satisfactory accuracy. Face detection using Viola-Jones algorithm based on trained cascade produced acceptable detection accuracy $(90.5 \pm 4.34 \%)$ with shortest detection time. The total average detection time for the VJ algorithm was about $23 \mathrm{~ms}$, so for the used platform it is theoretically faster to detect faces $(1 / 0.023=\sim 43 \mathrm{FPS})$ in video frames than to capture frames in the video acquisition mode ( 30FPS). The pattern-matching algorithm allowed to process one frame in average time of $42.8 \mathrm{~ms}$ with detection accuracy of $91.6 \pm 0.03 \%$ and the active-contour algorithm produced accuracy of $89.8 \pm 0.06 \%$ with longest detection time $(58.8 \mathrm{~ms})$.

The values of symmetry ratio show that this parameter depend on a subject that was examined. The face symmetry or head position may have influence on it. The PM algorithm was sensitive to template scaling. Even for the first level of the pyramid it was not able to detect the correct area. This problem could be solved by extracting template 
automatically from the first frame instead of defining it before algorithm execution. To do this, another method should be used to recognize a face. The detected area could be further used as a template for remaining frames. One of the considered method for detecting face in initial frames could be the VJ method, because the AC algorithm is not very useful since it produces many false positive results. This combination of $\mathrm{VJ}$ and PM algorithms could increase the precision of the system, as PM method allowed to achieve higher detection accuracy and it produced 0 false positives. On the other hand, it was much slower than VJ algorithm, therefore the method used should depend on the purpose which the system should achieve.

Taking into account the short processing time (especially for Viola-Jones algorithm) we believe that these methods could be used for detecting face in real-time in various medical applications. Some examples include the differential analysis of vascularization in the evaluation of facial paralysis or the estimation of the respiration rate using an analysis of temperature changes in the nostril region after extracting it and tracking [11]. Moreover, one can take advantage of recent growth in popularity of small thermal camera modules and embed them in smart glasses to examine a patient in real-time. Some examples include measuring respiration rate using thermal camera embedded in smart glasses and after the identification of a patient by using graphical markers [6] [25] or face recognition [8] storing results in the medical institution database.

In the framework of the eGlasses project we are developing the smart glasses platform that currently uses the OMAP Dual Core processor with $1.5 \mathrm{GHz}$ clock. Since hardware platforms are similar we hope that it will be possible to acquire thermal images and detect faces, practically in real time. Since the goal is to process video frames then additional frame-to-frame analysis of detected or not detected faces can be used for better final results.

To sum up, in this paper we described methods and results of a research on face detection algorithms in thermal images. As the aim of our work was to determine whether the computational performance of presented methods allow to process frames in real time, we measured time of processing one frame for different volunteers. The preliminary results show that best algorithm allows to detect face with acceptable accuracy $(90.5 \pm 4.34 \%)$ in about $23 \mathrm{~ms}$ per frame, what is acceptable for the needs of the smart glasses platform. Considering future work in this area, the algorithms should be examined in terms of sensitivity to movements performed by volunteer. If necessary, some steps should be taken for motion compensation in order to enhance the algorithm so that it can cope with very large head movements.

\section{REFERENCES}

[1] K. K. Sung and T. Poggio, "Example-based learning for view-based human face detection," IEEE Transactions on Pattern Analysis and Machine Intelligence, vol. 20, no. 1, pp. 39-51, Jan 1998., no. doi: 10.1109/34.655648.

[2] C. Chieh-Li and J. Bo-Lin, Infrared thermal facial image sequence registration analysis and verification, Infrared Physics \& Technology, vol. Volume 69, March 2015, pp. Pages 1-6.

[3] R. Lienhart and J. Maydt, "An Extended Set of Haar-like Features for Rapid Object Detection," USA: Intel Labs, Intel Corporation, Santa Clara, CA 95052,.

[4] D. Socolinsky and A. Selinger, "A comparative analysis of face recognition performance with visible and thermal infrared imagery," Proceedings of the 16th International Conference on Pattern Recognition, , 2002, vol. 4, 2002, , p. pp. $217-222$.

[5] A. Seal, S. Ganguly, D. Bhattacharjee, M. Nasipuri and D. K. Basu, "A Comparative Study of Human thermal face recognition based on Haar wavelet transform (HWT) and Local Binary Pattern (LBP)," Department of Computer Science and Engineering, Jadavpur University, Kolkata- 700032, India.

[6] A. Kwasniewska, J. Klimiuk-Myszk, J. Ruminski, J. Forrier, B. Martin and I. Pecci, "Quality of graphical markers for the needs of eyewear devices," in Human System Interactions (HSI), 2015 8th International Conference on , vol., no.,, pp. pp.388-395, 25-27 June 2015.

[7] J. Rumiński, M. Kaczmarek, A. Renkielska and Antoni Nowakowski, "Thermal Parametric Imaging in the Evaluation of Skin Burn Depth," IEEE TRANSACTIONS ON BIOMEDICAL ENGINEERING,, Vols. VOL. 54,, no. NO. 2,, FEBRUARY 2007.

[8] M. Smiatacz and J. Ruminski, "Local texture pattern selection for efficient face recognition and tracking," Proc. of the 9 International Conference on Computer Recognition Systems, CORES 2015, Advances in Intelligent Systems and Computing,, pp. pp. 359-368, Springer, 2015.

[9] M. Marzec, R. Koprowski and Z. Wróbel, "Methods of face localization in thermograms," Biocybernetics and Biomedical Engineering, Volume 35, Issue 2, Pages 138-146.

[10] F. Q. AL-Khalidi, R. Saatchi, D. Burke and H. Elphick, "Facial Tracking Method for Noncontact Respiration Rate Monitoring," (CSNDSP), 2010, pp. 751-754.

[11] F. Q. AL-Khalidi, R. Saatchi, D. Burke and H. Elphick, "Tracking Human Face Features in Thermal Images for Respiration Monitoring," (AICCSA), 2010, pp. 1-6.

[12] X. Liu, S. Dong, M. An, L. Bai and J. Luan, "Quantitative assessment of facial paralysis using infrared thermal imaging," 2015 8th International Conference on Biomedical Engineering and Informatics (BMEl), .

[13] S. Feng, S. K. Zhou, S. Good and D. Comaniciu, "Automatic fetal face detection from ultrasound volumes via 
learning 3D and 2D information," Computer Vision and Pattern Recognition 2009. CVPR 2009. IEEE Conference on, Miami, FL,

[14] P. Viola and M. Jones, " Robust Real-Time Face Detection,," Robust Real-Time Face Detection International Journal of Computer Vision 57(2), 2004.

[15] P. Menezes, J. C. Barreto and J. Dias, "Face tracking based on haar-like features and eigenfaces," IFAC/EURON Symposium on Intelligent Au- tonomous, 2004.

[16] J. Barreto, P. Menezes and J. Dias, "Human-robot interaction based on Haar-like features and eigenfaces," In:International Conference on Robotics and Automation, 2004.

[17] S. Shylaja, K. Murthy, S. Natarajan, N. Kumar and R. Agarwal, Effective Eye Localization using Local Binary Patterns, icwet(2):40-47, Ed., IJCA Proceedings on International Conference and workshop on Emerging Trends in Technology (ICWET 2012), March 2012.

[18] S. Wang, Z. Liu, P. Shen and Q. Ji, Eye localization from thermal infrared images, Pattern Recognition, vol. Volume 46, I. 10, Ed., October 2013, pp. Pages 2613-2621.

[19] E. Mostafa, R. Hammoud and A. F. A. Ali, "Face recognition in low resolution thermal images," Computer Vision and Image Understanding, vol. Volume 117, I. 1. Issue 12, Ed., December 2013, pp. Pages 1689-1694..

[20] P. Buddharaju, I. Pavlidis, P. Tsiamyrtzis and M. Bazakos, "Physiology-based face recognition in the thermal infrared spectrum," IEEE Trans. Pattern Anal. Mach. Intell. 29 (2007) 613-626.

[21] M. Marzec, R. Koprowski, Z. M. M. R. K. Wróbel, A. Kleszcz and A. Wilczyński, Automatic method for detection of characteristic areas in thermal face images., Multimedia Tools Appl. 74, 12 (June 2015), 4351-4368. .

[22] M. Marzec, R. Koprowski and Z. Wróbel, "Detection of selected face areas on thermograms with elimination of typical problems," Journal of medical informatics \& technologies Vol. 16/2010, Ed..

[23] K. S. Kumar, S. Prasad, V. B. Semwal and R. C. P. D. J. 2. Tripathi, "REAL TIME FACE RECOGNITION USING ADABOOST IMPROVED FAST PCA ALGORITHM," International Journal of Artificial Intelligence \& Applications; Jul 2011, Vol. 2 Issue 3, p45.

[24] S. Pramanik and D. Bhattacharjee, "Geometric feature based face-sketch recognition," Pattern Recognition, Informatics and Medical Engineering (PRIME), 2012 International Conference on, Salem, Tamilnadu, , pp. 2012, pp. 409-415.

[25] K. Czuszynski and J. J. Ruminski, "Interaction with medical data using QR-codes," in Human System Interactions (HSI), 2014 7th International Conference on, pp. pp.182-187, 16-18 June 2014. 